\title{
A COMPARISON OF ISMA AND DECT-DCS CHANNEL ACCESS POLICIES FOR INDOOR WIRELESS COMMUNICATIONS SYSTEMS
}

\author{
R. C. Atkinson \& J. Dunlop \\ Department of Electronic and Electrical Engineering \\ University of Strathclyde \\ Glasgow G1 1XW
}

\section{ABSTRACT}

This letter is concerned with the assessment of channel access mechanisms for indoor $3 \mathrm{G}$ mobile communications systems offering packet based services. One such service in which the channel access mechanism will have a significant impact is Voice Over Internet Protocol (VOIP). This letter considers the impact of two channel allocation schemes for the indoor environment: mobile-controlled dynamic channel selection (DCS), and basestation-controlled inhibit sense multiple access (ISMA). It is shown that the DCS scheme is subject to inferior performance in packet based voice transmission due to a higher degree of front end clipping which is introduced.

\section{INTRODUCTION}

The rapid growth in the cellular mobile market has surpassed expectations. As the number of users has increased there is a need to supply ubiquitous coverage, especially in the indoor arena. At present most indoor coverage is provided by buildings illuminated by external macrocell base stations. However, as the market continues to grow this approach may not meet the required capacity since active users, of all buildings within the beam-width of the BS antenna, must share the limited channels on the base station. A solution to this problem is the 
deployment of low power indoor picocellular base stations, to provide local coverage.

Operators have begun installation of GSM indoor base stations, and the DECT cordless technology has been well established in Europe for many years. It is widely expected that cellular and cordless systems will merge in future wireless communications systems and that indoor base stations will be required to support a wider range of services than their exterior counterparts. The channel access policies employed in such systems must be able to accommodate the requirements of the services which they support.

\section{ACCESS POLICIES}

The DECT system operates a DCS (Dynamic Channel Selection ) scheme whereby mobiles monitor the channel-set and transmit on a channel that they perceive to be unoccupied. The channel selection is based on a least interfered channel (LIC) algorithm which is designed to avoid the simultaneous use of a particular channel by more than one mobile. The operation of the LIC algorithm is satisfactory in a circuit switched environment, however this letter indicates that performance may not be satisfactory when supporting packet voice transmission.

Inhibit Sense Multiple Access (ISMA) has shown promise in the indoor environment due to its ability to resolve the hidden terminal problem. In contrast to the DECT system, the base station instructs each mobile on which channel to transmit, avoiding the potential of collision which may occur in the DECT case. 
A number of studies [1-3] have shown ISMA to provide high throughput values, however, its ability to carry voice traffic has not been fully examined.

\section{APPROACH}

Simulations have been conducted to compare the performance of the DCS and the ISMA schemes for voice traffic. The interior of a building was simulated, comprising of a number of rooms separated by internal walls. The Motley Indoor Propagation Model [4-6] was used to determine the signal power of the various mobiles at the basestation. The mobiles were uniformly distributed around the building, changing position periodically. A collision occurs if two or more mobiles attempt to transmit on the same channel. In this event one or more packets may be lost; depending on whether one mobile was able to capture the channel.

To enable proper comparison between the two techniques, a common speech frame length (20 ms) and frame structure ( 8 X 577 $\mu$ s) was used; the GSM system was deemed an appropriate model. Call traffic was generated via a poisson process (for equivalent circuit-switched traffic loads in erlangs). Throughout the duration of a call, voice activity is modelled by on-off periods of talkspurts and silences. During a talkspurt speech frames are created. Each speech frame is decomposed into speech packets and interleaved over four slots. An equivalent GSM system would interleave the speech frame over eight half-slots, however, in order to reduce end-to-end delay, it was decided that interleaving over four full slots was appropriate. In the evaluation of packet-speech systems two metrics are 
commonly used; $95^{\text {th }}$ percentile values for overall packet loss probability and packet loss probability at the beginning of talkspurts (packets lost from the lead speech frame constitute packet losses at the start of the talkspurt), shown in Figure 1. The metric values were extracted from the CDFs of various runs, averaged, and $95 \%$ confidence limits produced.

\section{RESULTS}

Figure 2 shows the average overall packet loss metric. It indicates similar performance of the two schemes, with ISMA providing superior performance below 20 erlangs, and DCS providing superior performance above this value.

When the packets are decomposed into separate groups: packets in middle/end of talkspurt and packets at start of talkspurt, a clearer picture of the relative performance of the two schemes emerges. Due to the greater number of packets in the middle/end of a talkspurt group, these packets dominate the overall packet loss metric. Figure 3 shows the relative performance of the two schemes considering packets lost in the middle/end of a talkspurt and shows similar to Figure 2 for both schemes.

Figure 4 shows the packet loss metric for packets lost at the start of a talkspurt, the ISMA scheme provides vastly superior performance compared to the DCS scheme.

Packets lost at the start of a talkspurt are more significant than those lost elsewhere; this is due to the brain's ability to interpolate missing information as a 
result of packet losses in the middle of a talkspurt, and therefore they have a greater impact in perceived speech quality.

\section{CONCLUSION}

This letter has examined two channel allocation schemes; mobile controlled (DCS scheme), and basestation controlled (ISMA scheme). It has been shown that the ISMA system provides superior speech quality than the DCS scheme. Whilst collisions that occur in circuit-switched operation of the DCS scheme are tolerable, the number of collisions becomes unacceptably high for packet access techniques. The high number of collisions is the result of repeated access attempts at the start of talkspurts.

\section{REFERENCES}

1. ZDUNEK, K. J., UCCI, D. R., LOCICERO, J. L.: 'Throughput of nonpersistent inhibit sense multiple access with capture', Electronics Letters, 1989, 25(1), pp. 30-32

2. PRASAD, N. R., NIJHOF, J. A. M., 'Indoor wireless communications using slotted non-persistent isma, 1-persistent isma and non-persistent ismalcd', Proc. IEEE Veh. Tech. Conf. (VTC-97), 1997, Vol. 3, pp. 1513-1517

3. PRASAD, R., LUI, C. Y., 'Throuput analysis of some mobile packet radio protocols in rician fading channels', IEE Proc.-I, 1992, 139(3), pp. 297-302

4. KEENAN, J. M., MOTLEY, A. J., 'Radio coverage in buildings', Br Telecom Technol. J., 1990, 8(1), pp. 19-24

5. MOTLEY, A. J., KEENAN, J. M. P., 'Personal communication radio coverage in buildings at $900 \mathrm{mhz}$ and $1700 \mathrm{mhz}$, Electronics Letters, 1988, 24(12), pp. 763-764

6. GOLLREITER, R., 'Channel models issue 2', ATDMA deliverable R2084/ESG/CC3/DS/P/029/b1, 1994, pp. 77-81 


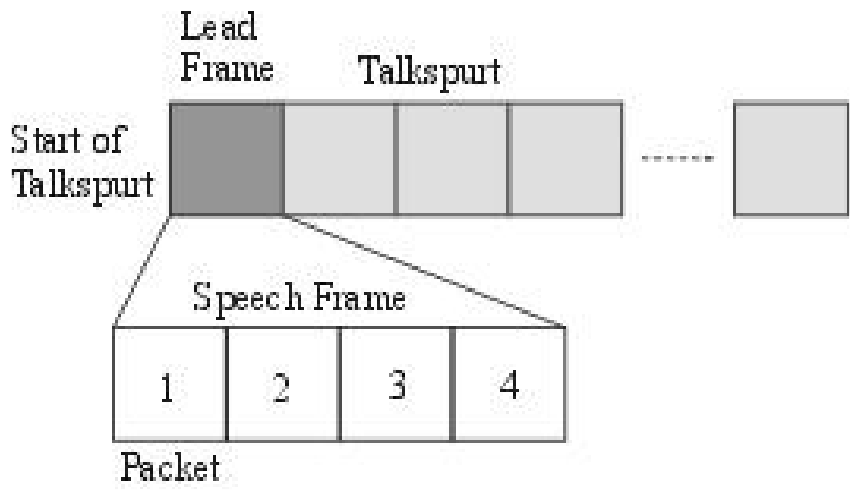

Figure 1 : Packets at start of talkspurt

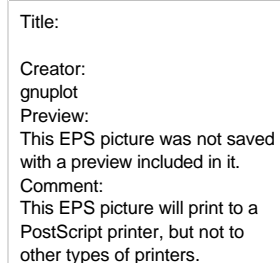

Figure 2 : Overall packet loss

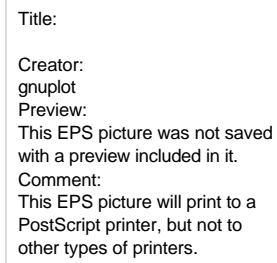

Figure 3 : Packet loss middle/end of talkspurt 
This EPS picture was not saved

with a preview included in it.

Comment:

This EPS picture will print to

PostScript printer, but not to

other types of printers.

Figure 4 : Packet loss start of talkspurt 